\title{
Maximising availability of transportation robots through intelligent allocation of parking spaces ${ }^{\star}$
}

\author{
Roopika Ravikanna, Marc Hanheide, Gautham Das, and Zuyuan Zhu \\ University of Lincoln, England
}

\begin{abstract}
Autonomous agricultural robots increasingly have an important role in tasks such as transportation, crop monitoring, weed detection etc. These tasks require the robots to travel to different locations in the field. Reducing time for this travel can greatly reduce the global task completion time and improve the availability of the robot to perform more number of tasks. Looking at in-field logistics robots for supporting human fruit pickers as a relevant scenario, this research deals with the design of various algorithms for automated allocation of parking spaces for the on-field robots, so as to make them most accessible to preferred areas of the field. These parking space allocation algorithms are tested for their performance by varying initial parameters like the size of the field, number of farm workers in the field, position of the farm workers etc. Various experiments are conducted for this purpose on a simulated environment. Their results are studied and discussed for better understanding about the contribution of intelligent parking space allocation towards improving the overall time efficiency of task completion.
\end{abstract}

Keywords: Robotic Farming · Agricultural Robots · Autonomous Parking · Robotic Fleets · Swarm Robotics

\section{Introduction}

Autonomous mobile robots have been extensively used to perform specific tasks in various application domains such as care homes, warehouses, and precision agriculture. In many of these environments, the tasks are dynamic, meaning they can appear at any time at any part of the environment, and the robots allocated to do these tasks should travel to one or more locations in the environment to execute the tasks. Most of these environments are structured, hence the path of the robot should be planned carefully in advance to reduce travelling time to these task locations. This work specifically addresses this challenge by dynamically allocating the parking spaces of robots closer to the area where demand is high. In particular, the deployment of a fleet of agricultural robots for in-field logistics operations to support human fruit pickers in a strawberry production poly-tunnel environment is considered here.

* Funded by the EPSRC Centre for Doctoral Training - AgriFoRwArdS, and Saga Robotics 


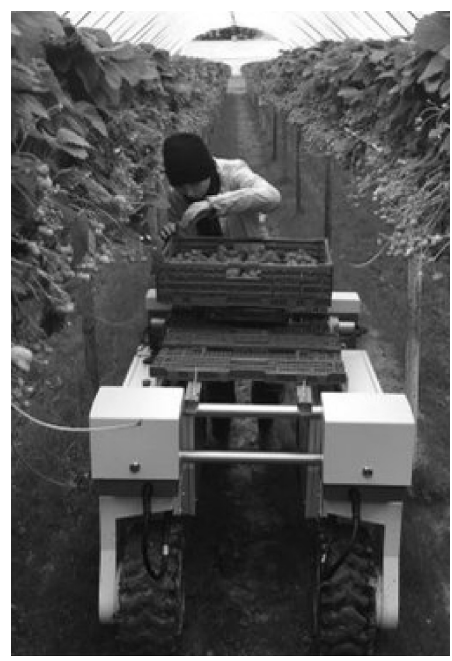

(a)

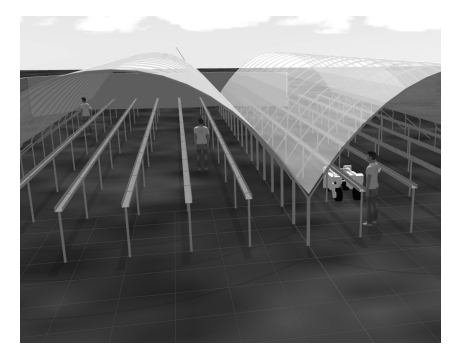

(b)

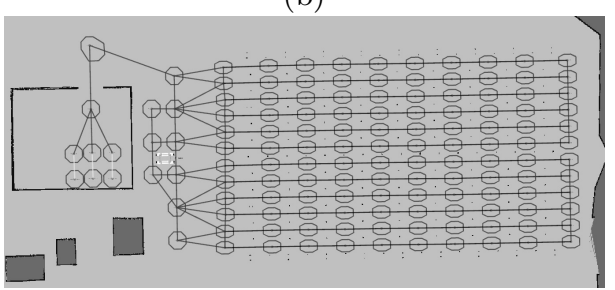

(c)

Fig. 1: (a) A representative image of a picker loading fruits into the robot; (b) Graphical representation of pickers in the rows of a poly-tunnel environment; and (c) Graphical representation of the field's Topological map.

The work we are presenting here is build upon our previous works in fleet coordination [3] and tracking of human in the field [7], with the aim of developing a robotic fleet that supports fruit pickers in soft-fruit production by automating transportation tasks, allowing the human pickers to focus on their job of picking. The overall system is readily deployed in various farm environments comprising autonomous Thorvald robotics platforms, coordinated by a central controller. A photograph of a picker performing loading of fruits is shown in Fig.1a. The feasibility study reported in [3] has shown that although the overall task completion time and hence the picking efficiency can be improved up to $20 \%$ by deploying a fleet of robots for fruit transportation, the pickers still have to wait for the robots after they request for one. This wait time increases when the robots have to travel longer distances to reach the picker. While in our previous work, the parking positions for robots (where they will wait for new transportation tasks to be allocated) in the farm environment was randomly fixed, in this work we focus on reducing the time pickers have to wait for a robot to serve them by proposing and evaluating novel approaches to dynamically reallocate these parking spaces. Hence, the contributions in this work are i) Novel approaches to dynamically reallocate robot waiting spaces to reduce the task start delay and overall task completion time and ii) Comprehensive experimental evaluations of the proposed approaches in discrete-event simulations (DES).

\section{Background and Related Work}

Agricultural robotics have been widely researched and deployed at different stages of food supply chain from fully autonomous precision field operations 
such as seeding, weeding and harvesting [4] to human-robot interactive applications such as in-field logistics [3]. With the background research maturing, many agri-robotic platforms targeting specific crops and applications are made commercially available [2]. Deploying a fleet of such robots is beneficial to distribute the tasks among the robots [5] and to improve the task completion. Specifically looking at in-field logistics operations, positioning the fleet of robots closer to the area of high demand can reduce the robots' travel time to the task locations as well improve the overall task completion metrics. This work explores this approach to dynamically allocate parking spaces for the robots.

The researchers in [6] claim that multi-robot task allocation can be reduced to an instance of the Optimal Assignment Problem. They perform a comparative study amongst popular task allocation strategies such as ALLIANCE, BLE amd $\mathrm{M}+$ to study differences in their computational complexity and impact on efficiency of task completion, which iterates the importance of strategies in task allocation towards maximising overall efficiency. The authors of [1] discuss in detail about a system of Unmanned Robotic Service Units in Agricultural tasks. Here, they point out that the three major problem areas in unmanned agricultural robots are their interaction with field workers, maneuvering and prioritisation of tasks. The problem of parking space allocation to robots can be considered vital towards enhancing the quickness of approach by the robots to farm workers, which in turn partly contributes to the improvement of the first mentioned problem area in robotised farming.

\section{Methodology}

For the purpose of our research, we discretise the spatial representation of the farm environment into a topological graph of nodes and edges. We assume human pickers travelling from node to node while picking, until they have exceeded the capacity of their picking crate and require a robot to take the picked fruits away and provide a new empty crate to continue picking into. Likewise, robots navigate along the topological graph, and we model their travel time along the edges, based on real-world parameters. Such a discretised representation of the problem, allows us to use the formalism of discrete event simulation (DES) to study the problem at hand [3].

DES models the operation of a multi-agent system as a discrete sequence of events in time and the basic unit agents, here, the human picker and the field robot, as entities [9]. The entities in the DES model compete among themselves for resources which are limited (e.g. in our case a node can only ever be occupied by single robot or picker, modelling the spatial constraints). Consequently when all robot are allocated to support pickers, any picker making a new request will have to wait in a queue to be allocated a robot. We use DES in our analysis as it allows to run simulations very fast, as any time between events (such as the waiting and travel times) discrete steps that do not occur in reality, allowing the efficient study of the proposed parking space allocation algorithms comprehensively. 
As described in our previous work [3], a gang of human pickers are assumed to pick berries in a strawberry production poly-tunnel environment with plants on raised tables in this work. Typically, the farm layout looks like a fork with a head lane along one open end of the tunnel and navigation rows between the tables inside the poly-tunnel, as shown in Fig.1b. Following our approach, a discrete topological map representation of the environment can easily capture the layout in which robots and pickers operate.A representation of the topological map of the poly-tunnel field is shown in Fig.1c. It is assumed that there is sufficient space along the header lanes to park multiple robots as well for other robots to pass through. Different approaches to dynamically assigning parking spaces along the header lane are proposed in the following.

\subsection{Parking Space Allocation Algorithms}

This paper suggests five different algorithms that help with the allocation of parking spots for robots in an agricultural setup. These are designed keeping in mind their need to be adaptable to different field sizes, number of pickers and the average time each picker takes for performing the picking action at each node before moving on to the next. The parking spot algorithms vary in their complexity of decision from random allocation to speed based cumulative ranking that takes into account factors including number of pickers, their position in the field, the average time they take up while picking etc. This is to observe if the allocation of parking spaces is indeed important to conserve resources and the global task completion time, and also if the performance improvement is consistent with the increasing complexity and intuitive intelligence of the parking allocation algorithms.

Random Ranking This is the simplest in design of all the suggested parking space allocation designs suggested. Of all of parking spaces spread across all row headers of the field, one is allocated at random to the robot irrespective of the size of the field, position of the pickers or their speed of picking. This algorithm is vital in providing a comparison of performance to all other parking space allocation algorithms. This is to firstly understand if there is in fact any positive consequence to providing intelligence to the task of parking space allocation. Fig.2c shows a demonstrative case of having implemented the Random Ranking technique. The figure shows 10 rows of crops and pickers located in rows $3,5,9,10$. Since Random Ranking is independent of any initial parameters of the field and pickers, it randomly generates row 2 to be the assigned parking space.

Middle Row Ranking Middle Row Ranking is built on the logic that a robot placed at a parking space near the center of the field would enable it to fairly access picker calls from any part of the field. In case of $\mathrm{n}$ robots, the robots shall be recommended to be parked sequentially in parking spaces at every $1 / n$th of the field. This is the second most simple design for parking space allocation suggested in this paper. Fig.2d shows a demonstrative case of having implemented the 
Middle Row Ranking technique. The figure shows 10 rows of crops and Pickers located in rows 3,5,9,10. Middle Row Ranking entirely bases its decision upon the width of the field, i.e. the number of rows in the field. Therefore, the row 5 , one of the centre rows is assigned as the parking space.

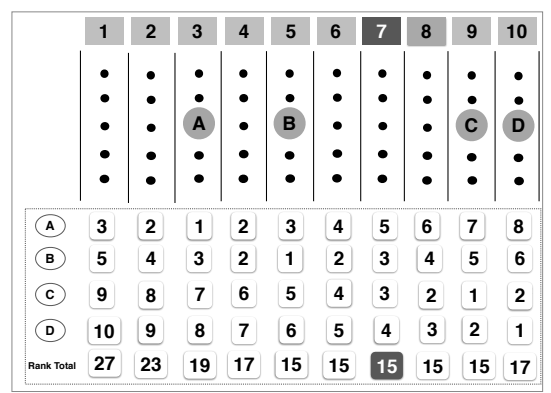

(a)

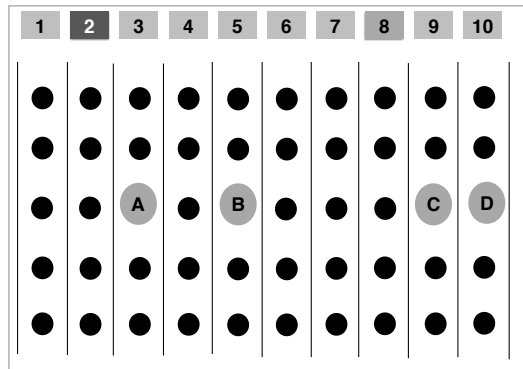

(c)

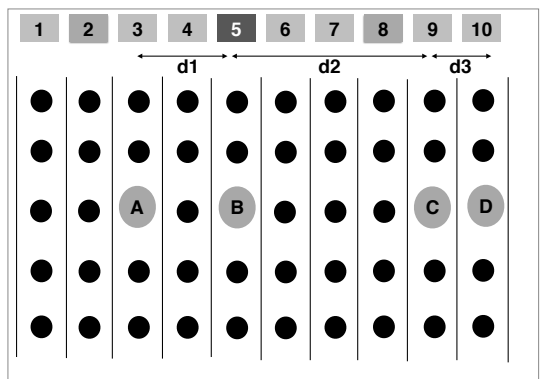

(e)

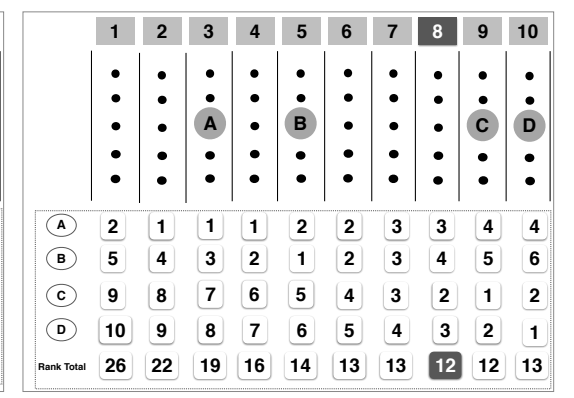

(b)
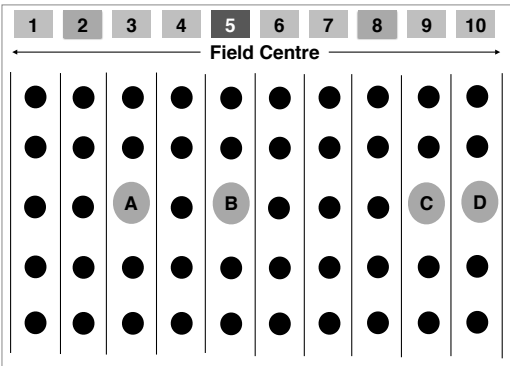

(d)

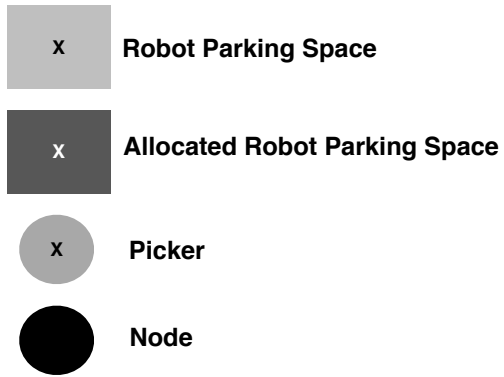

(f)

Fig. 2: Demonstrative Diagrams of (a) Cumulative Ranking, (b) Speed Based Cumulative Ranking, (c) Random Ranking, (d) Middle Row Ranking, (e) Distance Based Ranking are presented along with their reference legend in (f)

Distance Based Raking Distance Based Ranking looks at the distance in between each of the pickers in the field. The robot is allocated a parking space that lies in the approximate centre of the rows that indicate the largest gap 
between the pickers. The logic behind this algorithm is to tackle cases in which the concentration of pickers is to one side of the field rather than them being spread evenly across the field, which is the intuitive assumption made in the previously suggested Middle Row Ranking. Fig.2e shows a demonstrative case of having implemented the Distance Based Ranking technique. The figure shows 10 rows of crops and Pickers located in rows 3,5,9,10. This technique bases its decision upon the comparative distance in between the pickers. So despite the exact same initial conditions as discussed in the previous ranking technique Middle Row, the result through Distance Based Ranking varies choosing row 7 as the assigned parking space, which lies in the centre of the largest gap $d 2$, indicating the maximum distance between any two pickers in the given case.

Cumulative Ranking Cumulative Ranking makes a parking space allocation decision by aggregating the individual parking spot preferences given out by each of the pickers based on their position in the field. Fig.2a shows a demonstrative case of having implemented the Cumulative Ranking technique. It is seen in the figure that there are four Pickers A,B,C and D located in rows 3,5,9,10. The individual preferences assigned to rows by each of the pickers can be observed in the figure. Picker A gives out its preference of parking space allocation where, the space near its own row, row 3 is given the first priority with rank 1 and the rows that are subsequently adjacent are given with incrementally increasing ranks indicating a decreased preference to rows that are farther from the row of that particular picker. Pickers B, C and D do the same to all the rows of the field. The ranks given by each of the pickers for each of the rows are added up. The aggregated ranks of rows are now observed to find the least objected row, i.e. the row with the least rank number. The parking space near the header of this row is considered to be the most suitable one. In case of multiple rows holding the minimum rank, the median of that sequence of rows is taken as the winner. As a result of this, in this example row 7 which is the most mutually agreeable row amongst the pickers is assigned as the parking space.

Preferential Cumulative Ranking This is an extension from the Cumulative Ranking Technique but with inclusion of consideration given to the time taken by the individual pickers to move from one node to another i.e to perform the picking action. In simple terms, rankings given by the faster pickers are prioritised over that of the slower pickers. To enable this, the mean value of the set of times taken by each of the pickers to go from one node to another is calculated. Faster pickers are classified as those who take time less than or equal to the calculated mean value, those remaining are bracketed as the slower pickers. After this classification, the same procedure explained in Cumulative Ranking is carried out. The only difference is that, the slower pickers change their ranking preference for every two consecutive rows instead of one as in case of faster pickers. This is done in an attempt to mathematically reduce the implication of preference given by the slower pickers. Fig. $2 \mathrm{~b}$ shows a demonstrative case of having implemented the Preferential Cumulative Ranking technique. Here, Picker A which is a picker 
whose picking time is assumed to fall under the average picking time of all the pickers in the field is given a lesser preference that can be noticed through the change in the ranking priority it provides for the same case as in the Cumulative Ranking Technique. Therefore, while aggregating the preferences this time, row 8 is found to be the most agreeable row, it can be noted that row 8 is further away from the slow picker, Picker A than row 7 which was the calculated result without the preferential treatment in ranking.

\section{Experimental Evaluation and Results}

The performance of the parking space allocation algorithms mentioned in the methodology section are put through experimentation on a simple simulated environment. In order to keep the parametric values of the simulation as realistic as possible, the values defining the spacing of nodes in the farm, speed of the pickers while picking at each node, their capacity to hold on to yield before calling for a robot are derived through an approximation of the corresponding values used in [3] that has a similar experimental setup. This paper by itself uses verified empirical data obtained from real farms.

\subsection{Experimental setup}

In the simulated experimental setup the test environment is assumed to be a forked rectangular field with numerous parallel rows which have equally spaced nodes or way points that the pickers pass through in the course of their picking action. Based on data from [3], the length of each row is assumed to be 120 meters, the node to node distance in the field is assumed as 5 meters, thus creating 24 nodes in each row. There is a variable called 'picker time' that is used to indicate the time taken by a picker to go from one node to another, i.e. the time he spends picking at each node. This value is set at 2450 seconds. The robot is assumed to move at a speed of $1 \mathrm{~m} / \mathrm{s}$. This would imply that the robot takes 5 seconds to move from one node to another. As per the experiment the picker calls for the robot after having observed his collecting tray to be full. The rate at which he calls for the robot would differ based on how the yield of the crop/fruit is at every node. Based on the results and data observed in the experiments of [3], it is calculated that the picker approximately calls for the robot once every 7.7 nodes. So for this experiment, it is assumed that every picker calls for the robot once for every 8 nodes he traverses. All of the experimental results discussed below were aggregated over 20 randomised trials.

Comparison of Ranking Techniques by varying the number of rows The first experiment conducted is that of varying the width of the field by changing the number of rows (from 5 to 50) and holding the number of pickers on the field as constant (number of pickers $=3$ ). The performance of the different ranking strategies are shown in comparison to one another through the graphs in Fig.3. The two evaluation metrics observed are Global Task Completion Time, 
which is the total time taken for the task to complete. This is an indicator or the task completion efficiency. Another is the Robot Travel Time which is the Total Time for which the Robot has been in motion, this is an indicator of resource conservation.

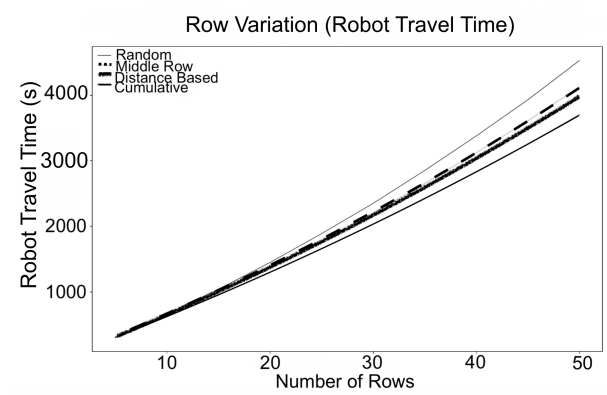

(a)

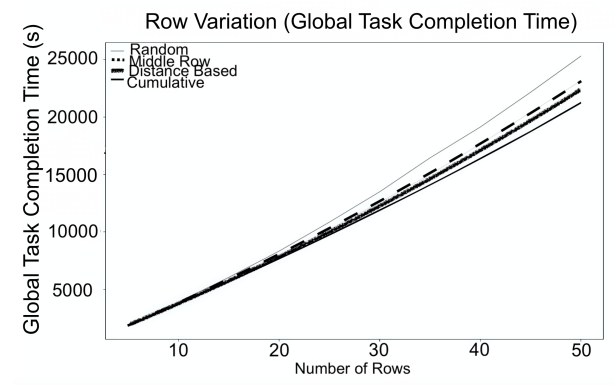

(b)

Fig. 3: Comparison of Performance of different ranking techniques by varying the number of rows from 5 to 50 and observing changes in (a) Robot Travel Time and (b) Global Task Completion Time

Comparison of Ranking Techniques by varying the number of pickers The next experiment conducted is that of varying the number of pickers (from 3 to 15 ) with a constant number of rows (number of rows $=100$ ) and the same picking speed as before. The performance of different ranking strategies for picker count variation are shown in comparison to one another through the graphs in Fig.4.

Impact of differences in Picking Time amongst Pickers The impact of differences in Picking Time amongst the Pickers is studied through this experiment. While assuming the number of Pickers to be 3 and varying the number of rows in the field, the performance of Cumulative Ranking is compared with that of Preferential Cumulative Ranking. It is thought that by giving a bias to faster pickers efficiency can be improved. Fig. 5 represents results from the experiment studying effects of change in speed of picking amongst pickers 


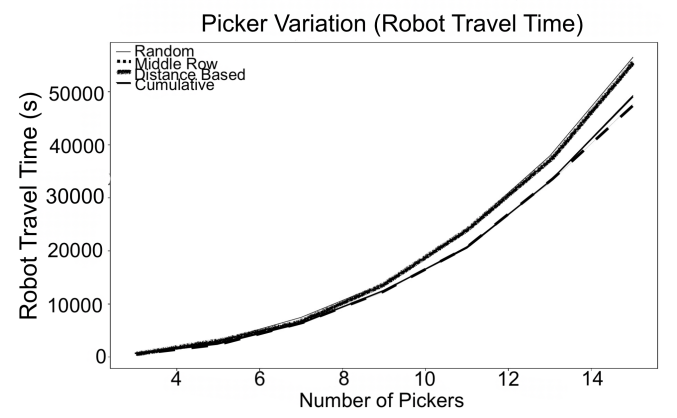

(a)

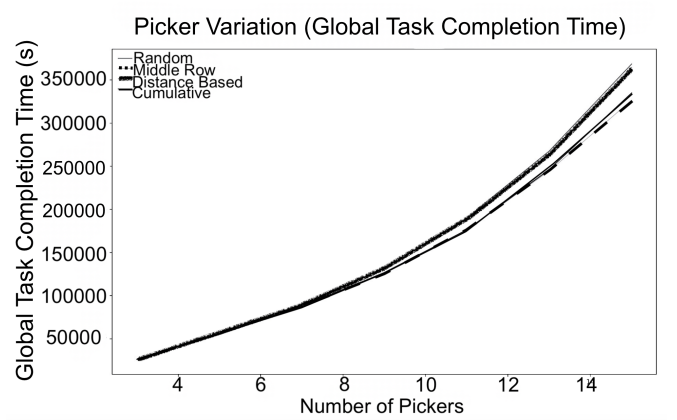

(b)

Fig. 4: Comparison of Performance of different ranking techniques by varying the number of pickers from 3 to 15 and observing changes in (a)Robot Travel Time and (b)Global Task Completion Time

\section{Discussions and Conclusion}

It can be observed from Fig 3, that in the experiment of comparing the ranking techniques through varying the number of rows, that the performance of the different ranking methods are proportional with respect to the two evaluation metrics. As expected, all remaining ranking strategies out perform Random Ranking, confirming the positive impact caused by intelligent planing of parking spaces. The best performer here is Cumulative Ranking, followed by Distance based Ranking and then Middle Row Ranking.

In Fig 4, showing the results of comparison of ranking techniques through varying the number of pickers, similar to the previous case the performance of the different ranking methods are proportional with respect to the two evaluation metrics. Here, it can be seen that with increase in the number of pickers for a fixed field size, Distance Based Ranking encounters a deterioration in performance only managing to be narrowly better than Random Ranking. This is because with the rise in number of pickers, the chances of a population skew of pickers over to one side of the field decreases, which was one of the main areas combated by the Distance Ranking methods. It can also be seen that Cumulative Ranking too begins to deteriorate with increase in the number of pickers, since the almost 


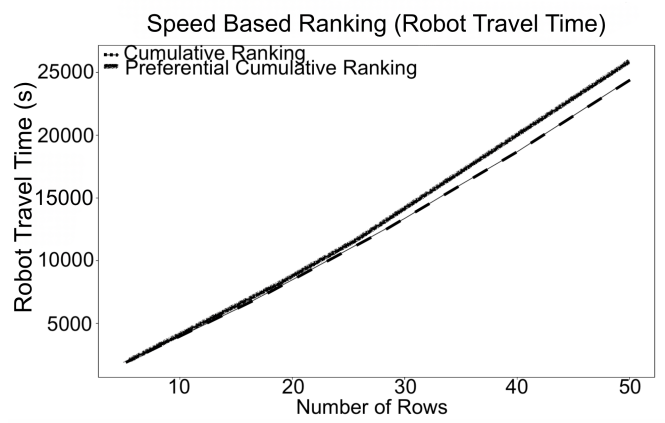

(a)

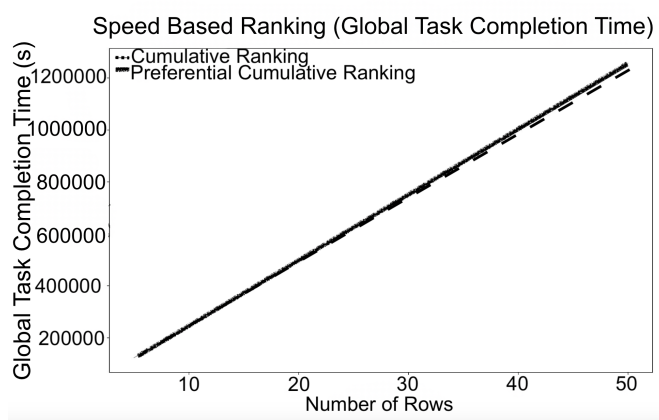

(b)

Fig. 5: Comparison of Performance of Cumulative and Preferential Cumulative Ranking techniques by varying the speed of picking amongst pickers and observing changes in (a) Robot Travel Time and (b) Global Task Completion Time

even distributions of the picker population might marginally favour one row over the other in case of multiple equally desired row priorities. This is why Middle Row Ranking works best with a High Pickers to Rows ratio, since there would most likely be equal demand for the robot from all areas of the field that would be best tackled by Middle Row Ranking.

In Fig 5 that represents results from the experiment studying effects of change in speed of picking amongst pickers, the results are different from what was hypothesised. The unbiased Cumulative Ranking out performs the speed based Preferential Cumulative ranking. This has been observed to be due to the following reason: though the robot might initially access the faster pickers quicker, when the demand for robot arises in the slower picker, the robot might have to for travel longer to reach this slow picker, this in turn increases the subsequent wait time of the faster pickers, slowing down the task. The results might however be different if in addition to the slowness, there is a reduction in demand from the slower pickers for access to the robots. Though this theoretically makes sense, it is highly unlikely that the changes in abilities and robot requirements of pickers working in the same field in a standard operation such as picking would produce such a case. Mathematically, it can be said that the constant of 
variation [8] in the picking speed and robot requirements of the pickers would not be high enough to trigger an impact due to them. Fig 5 shows the results from these experiments.

From the results obtained, it is noteworthy to observe that there is approximately a $\mathbf{2 0 \%}$ decrease in the Global Task Completion time and a $\mathbf{3 0 \%}$ decrease in robot usage when switching from a Randomised parking space allocation technique to adapting the Cumulative Ranking strategy. This proves the improvement to efficiency given by intelligent parking space allocation.

The results from the experiments performed on the inclusion of intelligence in parking space allocation for the waiting period of an autonomous agricultural robot show a positive impact on mechanical conservation in the use of robot due to reduced operational time and also a reduction in the global task completion time due to lower waiting periods by the pickers. Though all of the experiments shown in this paper demonstrate the case of a single robot, the methodologies can be easily extended to a system of multiple robots. There also lies interesting possibilities for the extension of these ideas to suit various field setups and farm shapes. These aspects of this research shall be addressed through continued work in the future.

\section{References}

1. Auat Cheein, F.A., Carelli, R.: Agricultural robotics: Unmanned robotic service units in agricultural tasks. IEEE Ind. Electron. Mag. 7(3), 48-58 (2013). https://doi.org/10.1109/MIE.2013.2252957

2. Bogue, R.: Fruit picking robots: has their time come? Ind. Robot Int. J. Robot. Res. Appl. (2020)

3. Das, G.P., Cielniak, G., From, P.J., Hanheide, M.: Discrete Event Simulations for Scalability Analysis of Robotic In-Field Logistics in Agriculture - A Case Study. In: ICRA 2018 Work. Robot. Vis. Action Agric. Brisbane (2018)

4. Duckett, T., Pearson, S., Blackmore, S., Grieve, B., Chen, W.H., Cielniak, G., Cleaversmith, J., Dai, J., Davis, S., Fox, C., Others: Agricultural robotics: the future of robotic agriculture. arXiv Prepr. arXiv1806.06762 (2018)

5. From, P.J., Grimstad, L., Hanheide, M., Pearson, S., Cielniak, G.: Rasberry-robotic and autonomous systems for berry production. Mech. Eng. 140(06), S14-S18 (2018)

6. Gerkey, B.P., Mataric, M.J.: Multi-robot task allocation: analyzing the complexity and optimality of key architectures. In: 2003 IEEE Int. Conf. Robot. Autom. (Cat. No.03CH37422). vol. 3, pp. 3862-3868 vol.3 (2003). https://doi.org/10.1109/ROBOT.2003.1242189

7. Khan, M.W., Das, G.P., Hanheide, M., Cielniak, G.: Incorporating spatial constraints into a bayesian tracking framework for improved localisation in agricultural environments. In: IEEE Int. Conf. Intell. Robot. Syst. pp. 2440-2445 (2020). https://doi.org/10.1109/IROS45743.2020.9341013

8. Pélabon, C., Hilde, C.H., Einum, S., Gamelon, M.: On the use of the coefficient of variation to quantify and compare trait variation. Evol. Lett. 4(3), 180-188 (2020)

9. Schriber, T.J., Brunner, D.T., Smith, J.S.: Inside discrete-event simulation software: How it works and why it matters. In: Proc. Winter Simul. Conf. 2014. pp. 132-146 (2014). https://doi.org/10.1109/WSC.2014.7019884 